TRANSACTIONS OF THE

AMERICAN MATHEMATICAL SOCIETY

Volume 300, Number 2, April 1987

\title{
ON BOUNDED ANALYTIC FUNCTIONS IN FINITELY CONNECTED DOMAINS
}

\author{
ZBIGNIEW SLODKOWSKI
}

\begin{abstract}
A new proof of the corona theorem for finitely connected domains is given. It is based on a result on the existence of a meromorphic selection from an analytic set-valued function. The latter fact is also applied to the study of finitely generated ideals of $H^{\infty}$ over multiply connected domains.
\end{abstract}

Introduction. In this paper, which is a direct continuation of [18], we study applications of analytic multifunctions to some topics in function theory on finitely connected domains, related mainly to the corona problem.

Concerning analytic multifunctions (which are certain set-valued functions, cf. Definition 1.2), $\S \S 1-3$ of $[\mathbf{1 8}]$ form sufficient background for our purposes here. The reader is also referred there for the information about the origin of the approach employed in this paper. However, basic definitions and references are provided below.

Our basic new result (Theorem 1.4) associates to every analytic multifunction defined in a finitely connected domain some meromorphic functions with poles at the critical points of Green's function of this domain. (Some improvements of this fact, in the special case of an annulus, are discussed in $\S 3$.)

This main technical result is applied in $\S 2$ to help obtain a new, simple proof of the well-known corona theorem for finitely connected domains. A novel feature of this proof (as compared with e.g. Forelli [9], Stout [19], Gamelin [7]) is that it does not use the corresponding result for the unit disc.

The same methods are used in $\S 4$, where we consider the question, when some power of $g$ from $H^{\infty}(G)$ belongs to the ideal $A f_{1}+A f_{2}+\cdots+A f_{n}$. We obtain generalizations to multiply connected domains of a result due to $\mathrm{T}$. Wolff (Theorems 4.1 and 4.2), as well as its refinements in the case of the unit disc (Corollary 4.4 and Example 5.3).

All proofs given in this paper extend to finite Riemann surfaces.

1. A meromorphic selection theorem. The main result of this section relates some meromorphic vector-valued functions to analytic multifunctions defined in finitely connected domains. First, we recall some definitions.

DEFINITION 1.1 (FOLK). A locally compact set $Z \subset \mathbf{C}^{n}$ is a maximum set if for every compact set $N \subset Z$ and for every analytic function $f$ defined in a neighborhood of $N$ the inequality $\max _{N}|f| \leq \max _{\partial_{Z} N}|f|$ holds.

DEFINITION $1.2[\mathbf{1 6}, \mathbf{1 8}]$. An upper semicontinuous set-valued correspondence $z \rightarrow K(z): G \rightarrow 2^{\mathbf{C}^{n}}$, where $G \subset \mathbf{C}$ is open and all $K(z)$ are nonempty and

Received by the editors January 16, 1986. Presented to the Society, Special Session on Convex Analysis, New Orleans, January 11, 1986.

1980 Mathematics Subject Classification (1985 Revision). Primary 32A80, 32E20, 46J15. 
compact, is analytic if the graph of $K$ has maximum property, where

$$
\operatorname{gr}(K)=\left\{(z, w) \in G \times \mathbf{C}^{n}: w \in K(z)\right\} .
$$

We need also some basic facts from the function theory on finitely connected domains. Our reference is Fisher [4, Chapter 4]. We consider only finitely connected planar domains $G$ whose boundary $\partial G$ consists of $(k+1)$ simple closed analytic curves. For a fixed point $p$, let

$$
z_{1}^{*}, \ldots, z_{k}^{*} \text { be the critical points of } g(z ; p),
$$

where $g(z ; p)$ is the Green's function of $G$ with a pole at $p$, and let $d \omega_{p}$ denote the corresponding harmonic measure. Set

$$
P(z)=\left(z-z_{1}^{*}\right)\left(z-z_{2}^{*}\right) \cdots\left(z-z_{k}^{*}\right) .
$$

We denote by $A(G)$ the algebra of all functions analytic in $G$ and continuous in $\bar{G}$, and by $H^{\infty}(G)$ the algebra of all bounded analytic functions on $G$. Because of the smoothness of $\partial G$, the nontangential boundary values $\tilde{f}(\varsigma)$ exist a.e. $d \omega_{p}$. We will use the following theorem (see e.g. proof in [4, Chapter IV, Theorem 4.8 and Proposition 4.2].

THEOREM $1.3[\mathbf{1 3}, \mathbf{2 0}]$. Let $f \in L^{\infty}\left(\partial G, d \omega_{p}\right)$. Then

$$
0=\int_{\partial G} f(\varsigma) \tilde{h}(\varsigma) d \omega_{p}(\varsigma), \quad h \in A(G), h(p)=0,
$$

if and only if there is an $F \in H^{\infty}(G)$, such that $f=\tilde{F} / P$ a.e. $d \omega_{p}$ on $\partial G$.

We can formulate now the main theorem of this section.

THEOREM 1.4. Let $G$ be a finitely connected domain with smooth real-analytic boundary and let $L: \bar{G} \rightarrow 2^{\mathbf{C}^{n}}$ be an upper semicontinuous multifunction such that its restriction to $G$ is analytic and all its sections $L(z), z \in \bar{G}$, are convex. Fix $p \in$ $G$. Then for every $w^{*} \in L(p)$, there exists a vector-valued function $F \in H^{\infty}\left(G, \mathbf{C}^{n}\right)$, such that $W(p)=w^{*}$ and $\tilde{W}(\varsigma) \in L(\zeta)$ a.e. $d \omega_{p}$, where $W(z)=F(z) / P(z)$, $z \neq z_{1}^{*}, \ldots, z_{k}, z \in G$.

PROOF. Using the multifunction $L(\cdot)$, we define a new norm $\|\cdot\| \|$ on $L^{1}=$ $L^{1}\left(\partial G, \mathbf{C}^{n+1}\right)$, equivalent to the usual norm

$$
\|f\|_{1}=\left\|\left(f_{0}, f_{1}, \ldots, f_{n}\right)\right\|_{1}=\sum_{i=0}^{n} \int_{\partial G}\left|f_{i}(\varsigma)\right| d \omega_{p}(\varsigma),
$$

and construct a linear functional on $L^{1}$ of norm one with respect to the norm $\||\cdot|\|$. $W(\cdot)$ will be determined in terms of an $L^{\infty}\left(\partial G, \mathbf{C}^{n+1}\right)$ function representing this functional.

To define the new norm, fix $r \in(0,1)$ and set for every $z \in \bar{G}$ :

$$
B(z)=\operatorname{co}\left(B_{n+1}(0, r) \cup\left\{\left(e^{i \theta}, e^{i \theta} w\right): 0 \leq \theta<2 \pi, w \in L(z)\right\}\right),
$$

(where $B_{n+1}(0, r)$ denotes the ball of radius $r$ ). Clearly $B(z), z \in \bar{G}$, are norming bodies in $\mathbf{C}^{n+1}$. Moreover,

$$
B(z) \cap\left(\{\operatorname{Re} \mu \geq 1\} \times \mathbf{C}^{n}\right)=\{1\} \times L(z) .
$$


Since $B(z), z \in \bar{G}$, are uniformly equivalent to the unit ball of $\mathbf{C}^{n+1}$, the formula

$$
|||f| \|=\int_{\partial G} \max \{|\langle w, f(\varsigma)\rangle|: w \in B(\zeta)\} d \omega_{p}(\zeta),
$$

where $\langle w, v\rangle=\left(w_{0} v_{0}+w_{1} v_{1}+\cdots+w_{n} v_{n}\right)$ defines a norm equivalent to the norm (1.3).

Let $A\left(G, \mathbf{C}^{n+1}\right)$ denote the (nonclosed) subspace of $L^{1}\left(\partial G, \mathbf{C}^{n+1}\right)$ consisting of all $f=\left(f_{0}, f_{1}, \ldots, f_{n}\right)$, such that $f_{i} \in A(G), i=0, \ldots, n$. Define a linear form $\Phi_{0}$ on $A\left(G, \mathbf{C}^{n+1}\right)$ by the formula

$$
\Phi_{o}(f)=\left\langle w^{\prime}, f(p)\right\rangle, \quad \text { where } w^{\prime}=\left(1, w^{*}\right) .
$$

We will show that $\Phi_{0}$ has norm one relative to $\|\cdot|\||$.

Consider $v(z):=\max \{|\langle w, f(z)\rangle|: w \in B(z)\}=\max \left(v_{1}(z), v_{2}(z)\right)$, where

$$
\begin{aligned}
& v_{1}(z)=\max \{|\langle w, f(z)\rangle|: w \in\{1\} \times L(z)\}, \\
& v_{2}(z)=\max \{|\langle w, f(z)\rangle|:|w| \leq r\}=r|f(z)|,
\end{aligned}
$$

and $f \in A\left(G, \mathbf{C}^{n+1}\right)$. The function $v_{1}(\cdot)$ is subharmonic by [18, Proposition 3.4] (use the plurisubharmonic function $u\left(z, w_{1}, \ldots, w_{n}\right)=\left|\left\langle\left(1, w_{1}, \ldots, w_{n}\right), f(z)\right\rangle\right|$ and the analyticity of $L(\cdot))$. Clearly $v_{2}(\cdot)$ is subharmonic, and so is $v(\cdot)$. By the mean-value inequality with respect to harmonic measure $d \omega_{p}$, we obtain for every $f \in A\left(G, \mathbf{C}^{n+1}\right)$

$$
\left|\Phi_{0}(f)\right|=\left|\left\langle w^{\prime}, f(p)\right\rangle\right|=v(p) \leq \int_{\partial G} v(\varsigma) d \omega_{p}(\varsigma)=\|\mid f\| \| .
$$

By the Hahn-Banach theorem, $\Phi_{0}$ has a $\mathbf{C}$-linear extension $\Phi$ to $\left(L^{1}\left(G, \mathbf{C}^{n+1}\right)\right.$, $\||\cdot|||)$, such that $\||\Phi|\| \leq 1$. Therefore there exists a unique vector-valued function $W^{\prime}=\left(W_{0}, \ldots, W_{n}\right)$, where $W_{i} \in L^{\infty}(\partial G)$ and

$$
\Phi(f)=\int_{\partial G}\left\langle W^{\prime}(\zeta), f(\zeta)\right\rangle d \omega_{p}(\zeta),
$$

for all $f \in L^{1}\left(\partial G, \mathbf{C}^{n+1}\right)$.

The property $\|\mid \Phi\| \| \leq 1$ implies that $W^{\prime}(\varsigma) \in B(\varsigma)$ a.e. $d \omega_{p}$. To check this, choose a countable dense set $\left\{v_{s}\right\} \subset \mathbf{C}^{n+1}$, and consider all points $\varsigma^{*} \in \partial G$, which are points of essential continuity (relative to $d \omega_{p}$ ) for each of the following functions:

$$
\begin{aligned}
& \varsigma \rightarrow W_{i}(\varsigma), \quad i=0,1, \ldots, n \\
& \varsigma \rightarrow \max \left\{\left|\left\langle w, v_{s}\right\rangle\right|: w \in B(\varsigma)\right\} .
\end{aligned}
$$

Seeing that the set of all such points $\varsigma^{*}$ has measure one, it suffices to show that $W^{\prime}\left(\varsigma^{*}\right) \in B\left(\varsigma^{*}\right)$. Suppose it does not; then there is a vector $v_{s} \in B\left(\varsigma^{*}\right)^{\circ}$ (= the polar of $\left.B\left(\varsigma^{*}\right)\right)$, such that $\left|\left\langle W^{\prime}\left(\varsigma^{*}\right), v_{s}\right\rangle\right|>1$. Consider arcs $A(t) \subset \partial G$, with center at $\zeta^{*}$ (relative to the arc-length measure) and such that $\lim _{t} \omega_{p}(A(t))=0$. Let

$$
f_{t}(\varsigma)=\left(\omega_{p}(A(t))\right)^{-1} 1_{A(t)} v_{s}
$$

Then, by the essential continuity at $\varsigma^{*}$,

$$
\lim _{t}||\left|f_{t}\right||| \leq 1, \quad \lim _{t} \Phi\left(f_{t}\right)=\left|\left\langle F\left(\varsigma^{*}\right), v_{s}\right\rangle\right|>1,
$$

which contradicts $\||\Phi|\| \leq 1$. 
Applying (1.6) and (1.7) to $f(\varsigma) \equiv(1,0, \ldots, 0)$, we obtain

$$
1=\int_{\partial G} W_{0}(\varsigma) d \omega_{p}
$$

and because $\left|W_{0}(\zeta)\right| \leq 1$ a.e. (since $W^{\prime}(\varsigma) \in B(\varsigma)$ a.e.), we get $W_{0}(\varsigma)=1$ a.e. By this and (1.4), we conclude

$$
\left(W_{1}(\varsigma), \ldots, W_{n}(\varsigma)\right) \in L(\varsigma) \quad \text { a.e. } d \omega_{p}
$$

We will show now that $W(\varsigma):=\left(W_{1}(\zeta), \ldots, W_{n}(\zeta)\right)$ has the required meromorphic extension to $G$. By $(1.6), \Phi(f)=0$, if $f \in A\left(G, \mathbf{C}^{n+1}\right)$ and $f(p)=0$, and by (1.7), for $i=1, \ldots, n$,

$$
0=\int_{\partial G} f(\zeta) W_{i}(\zeta) d \omega_{p}(\zeta), \quad \text { for all } f \in A(G) \text { with } f(p)=0
$$

By Theorem 1.3, $W_{i}(\varsigma)=\tilde{F}_{i}(\varsigma) / P(\varsigma), i=1, \ldots, n$, with $F_{i} \in H^{\infty}(G)$, and $W(z)=$ $(1 / P(z))\left(F_{1}(z), \ldots, F_{n}(z)\right)$ is the required meromorphic extension.

It remains to show, that $W(p)=w^{*}$. Substituting functions of the form $f(\varsigma)=$ $(0, \ldots, 0,1,0, \ldots, 0)$ to $(1.6)$ and $(1.7)$, we get

$$
w_{i}=\int_{\partial G} W_{i}(\varsigma) d \omega_{p}(\varsigma), \quad i=1, \ldots, p
$$

Integration against harmonic measure $d \omega_{p}$ is equivalent to integration (along the boundary) of the differential $(-1 / 2 \pi i) d g(z ; p)$. Since the latter differential has simple pole with residue one at $p$ and zeros at the critical points $z_{1}^{*}, \ldots, z_{k}^{*}$, therefore by the residue formula, the integral (1.8) is equal to $W_{i}(p)$ and so $W(p)=w^{*}$, as required. (See $[\mathbf{4}$, pp. 92-93] for more details.) Q.E.D.

An alternative proof. It may be of interest to the reader, that Theorem 1.4 can be also proven by extending the methods of Alexander and Wermer $[\mathbf{1}, \S 1]$. (This was actually the first proof we found.) Below, we indicate the necessary modifications, leaving to the reader their actual implementation into the proof of Theorem 1 in [1].

Let $A$ be the uniform closure, on the graph of $L$, of all rational functions in $z, w_{1}, \ldots, w_{n}$ without singularities on $\operatorname{gr}(L)$. The evaluation functional $f \rightarrow$ $f\left(p, w^{*}\right): A \rightarrow \mathbf{C}$ has a Jensen representing measure $d m$ supported on

$$
Y=\{(\varsigma, w): \varsigma \in \partial G, w \in L(\varsigma)\}
$$

that is

$$
\log \left|f\left(p, w^{*}\right)\right| \leq \int \log |f| d m, \quad f \in A .
$$

As in Alexander and Wermer $[\mathbf{1}]$, measure $d m$ can be disintegrated with respect to the projection $(\varsigma, w) \rightarrow \varsigma$, so that for a.a. $\varsigma$ in $\partial G$ (with respect to $d m^{*}=$ the projection of $d m$ ), we obtain a probability measure $d \sigma_{\varsigma}$ on $L(\varsigma)$, such that

$$
\int_{Y} f d m=\int_{\partial G} d m^{*} \int_{L(\varsigma)} f d \sigma_{\zeta}, \quad f \in C(Y) .
$$

Applying (1.9), (1.10) to functions $f(z, w)$, independent on $w$, we obtain, that $d m^{*}$ is the Jensen representing measure for $A(G)$, and so is equal to $d \omega_{p}$. Define finally

$$
W(\varsigma)=\int_{L(\varsigma)} w d \sigma_{\varsigma}
$$


The required properties of $W(\zeta)$ are checked mostly as in [1], and the modifications necessitated by the presence of critical points $z_{1}^{*}, \ldots, z_{k}^{*}$ are handled with the use of Theorem 1.3, similarly as in the proof given above.

REMARKS. The classical F. and M. Riesz theorem has been generalized to the context of finite Riemann surfaces by J. Wermer [20, Theorem 1]. A related result for finitely connected planar domains was obtained by W. Rudin [13, Corollary 3.6]. Theorem 1.3 is a special case of these results.

Theorem 1.4 generalizes a result on structure of polynomial hulls with convex sections obtained independently by Alexander and Wermer [1] and the author [17]. The latter result was applied to analytic multifunctions by Berndtsson and Ransford [2, Theorem 1.3]. Similarly, we formulate Theorem 1.4 for analytic multifunctions, rather than for rational hulls.

We would like to point out, that the proof of Theorem 1.4 is new also in the special case of the unit disc (compare with [17]).

2. A new proof of the corona theorem for finitely connected domains. In this section we prove the following lemma, from which the corona theorem for finitely connected domains follows immediately.

LEMMA 2.1. Let $G$ be a finitely connected planar domain as in Theorem 1.4 and let $z_{1}^{*}, \ldots, z_{k}^{*}$ be the critical points of Green's function with respect to $p \in G$. Let $\delta>0$ and $f_{1}, \ldots, f_{n} \in H^{\infty}(G)$ be given, such that $\delta^{2}<\left|f_{1}(z)\right|^{2}+\cdots+\left|f_{n}(z)\right|^{2}<1$, $z \in G$. Assume, that there are functions $k_{1}, \ldots, k_{n} \in H^{\infty}(G)$, such that

$$
\sum_{i} k_{i}\left(z_{j}^{*}\right) f_{i}\left(z_{j}^{*}\right)=1, \quad j=1, \ldots, k,
$$

and

$$
\sup _{z} \sum_{i}\left|k_{i}(z)\right|^{2} \leq M^{2}
$$

Then, there exist $g_{1}, \ldots, g_{n} \in H^{\infty}(G)$, such that $\sum_{i} f_{i} g_{i} \equiv 1$ in $G$ and

$$
\sup _{z}\left(\sum_{i}\left|g_{i}(z)\right|^{2}\right)^{1 / 2} \leq(1+M)\left(1+C_{\delta}\right)
$$

where $C_{\delta}$ is a constant depending only on $\delta$.

Let us recall that a multifunction $K: G \rightarrow 2^{\mathbf{C}^{n}}$, where $G \subset \mathbf{C}^{k}$ is open, is analytic $[\mathbf{1 6}, \S 2 ; \mathbf{1 8}$, Introduction], if $K$ is compact-valued, upper semicontinuous, and for every complex hyperplane $L \subset \mathbf{C}^{k+n}$ with $\operatorname{dim} L=n+1$, the intersection $L \cap \operatorname{gr}(K)$ has the maximum property in the sense of Definition 1.1. We need the following facts about analytic multifunctions.

Proposition 2.2. If $G_{1} \subset \mathbf{C}, G \subset \mathbf{C}^{k}, K: G \rightarrow 2^{\mathbf{C}^{k}}$ is an analytic multifunction and $f: G_{1} \rightarrow G$ is an analytic vector-valued function, then the composition $L(z)=K(f(z))$ is an analytic multifunction in $G_{1}[15$, Proposition 5.1(ii); 18, Lemma 2.4, Remark 3.7].

THEOREM $2.3[15$, Theorem 4.3 , case $n=2 ; 18, \S 1]$. Let $0<\delta<r$ and $P_{\delta, r}=\left\{z \in \mathbf{C}^{n}: \delta<|z|<r\right\}$. Then there exists an upper semicontinuous 
multifunction $K: \bar{P}_{\delta, r} \rightarrow 2^{\mathbf{C}^{n}}$, analytic in $P_{\delta, r}$ such that $K(z) \subset\left\{w \in \mathbf{C}^{n}:\langle w, z\rangle=\right.$ 1\}, $z \in \bar{P}_{\delta, r}$, where $\langle z, w\rangle=z_{1} w_{1}+\cdots+z_{n} w_{n}$. Moreover

$$
\max \left\{|w|: w \in K(z), z \in \bar{P}_{\delta, r}\right\} \leq C_{\delta, r},
$$

where $C_{\delta, r}$ is independent on $n$.

We will frequently make use of the following observation.

REMARK 2.4. If $G$ is any domain regular in the sense of potential theory (finitely or infinitely connected), it can be exhausted by domains of the form $G_{\varepsilon}=$ $\{z \in G: g(z, p)>\varepsilon\}$ with smooth analytic boundaries. In this case, a problem in question consists in giving uniform estimates (like in the next proof), the above observations allow us to reduce it to the case, when domain $G$ is finitely connected and has smooth boundary, and functions forming data of the problem are smooth on $\bar{G}$.

ProOF OF LEMMA 2.1. We can represent

$$
1=\sum_{i} k_{i}(z) f_{i}(z)+P(z) q(z), \quad z \in G,
$$

where $P$ is given by (1.2) and $q \in H^{\infty}(G)$. Clearly,

$$
\|P q\|_{\infty} \leq 1+M \text {. }
$$

By Remark 2.4, we can assume that functions $f_{1}, \ldots, f_{n}$ are smooth on $\bar{G}$ and $\partial G$ is smooth and real analytic.

Let $K: \bar{P}_{\delta, 1} \rightarrow 2^{\mathrm{C}^{n}}$ be as in Theorem 2.3. By Proposition 2.2, the composition $L(z)=K(f(z))$ is a uniformly bounded analytic multifunction, upper semicontinuous in $\bar{G}$. By Theorem 1.4, there exists $F=\left(F_{1}, \ldots, F_{n}\right) \in H^{\infty}\left(G, \mathbf{C}^{n}\right)$, such that $\tilde{W}(\varsigma) \in L(\varsigma)$ a.e. $d \omega_{p}$, where $W(z)=(1 / P(z)) F(z), z \in G, z \neq z_{1}^{*}, \ldots, z_{k}^{*}$. In particular, $\langle\tilde{W}(\varsigma), f(\varsigma)\rangle=1$ a.e. $d \omega_{p}$. Let

$$
g_{i}(z)=k_{i}(z)+F_{i}(z) q(z), \quad i=1, \ldots, k .
$$

Then $g_{i} \in H^{\infty}(G)$, and $\sum_{i} f_{i} g_{i} \equiv 1$ in $G$, for

$$
P(\varsigma) q(\varsigma) \cdot 1=P(\varsigma) q(\varsigma)\langle\tilde{W}(\varsigma), f(\varsigma)\rangle=\langle q(\varsigma) \tilde{F}(\varsigma), f(\varsigma)\rangle .
$$

Furthermore,

$$
\begin{aligned}
\sup _{z \in G} \sum_{i}\left|F_{i}(z) q(z)\right|^{2} & \leq \sup _{\zeta \in \partial G} \sum_{i}\left|\tilde{W}_{i}(\varsigma) P(\varsigma) q(\varsigma)\right|^{2} \\
& \leq\left(\sup _{\varsigma} \sum_{i} \mid \tilde{W}_{i}(\varsigma) \|^{2}\right)\|P q\|_{\infty}^{2} \leq C_{\delta, 1}^{2}(1+M)^{2},
\end{aligned}
$$

by (2.5) and (2.4). This and (2.2) gives (2.3). Q.E.D.

REMARK. The last proof works with minimal changes for finite Riemann surfaces (instead of using polynomial $P$, just observe, that $(1-\langle k, f\rangle W)$ is regular in $G)$. Theorem 1.4 would have to be reformulated in the context of finite Riemann surfaces. Its present proof does not require any modifications, except for the use of a general version of Theorem 1.3, due to Wermer [20] and Royden [12]. We omit further details. 
COROLLARY 2.5. If $G$ is an arbitrary domain, regular in the sense of potential theory and such that the sequence of critical points of $G$ is an interpolation sequence for $H^{\infty}(G)$, then the corona problem has solution in $G$.

ProOF. Apply Lemma 2.1 and Remark 2.4, use the interpolation property to find $k_{i}$ in (2.1); the interpolation constant can serve as $M$ in (2.2).

REMARKS. The corona theorem for finitely connected domains on Riemann surfaces is a classical result, which was proved and reproved many times; see, e.g. Forelli [5], Gamelin [7], Stout [19].

Lemma 2.1 and Corollary 2.5 are due to Jones and Marshall [9] (as well as Remark 2.4). However, the proof given above is different from theirs.

The first application of analytic multifunctions to the corona problem was found by Berndtsson and Ransford, who obtained a new proof of T. Wolff's $\bar{\partial}$-theorem. This approach was followed by Slodkowski [18], and further continued by Berndtsson and Ransford [2].

The proof given here generalizes the earlier one, presented in [18], which was a direct proof (without use of the $\bar{\partial}$-equation) of the corona theorem for the disc. (Another direct proof was given by Berndtsson and Ransford [2].)

3. Selection theorem for the annulus. If $G$ is an annulus, Theorem $1.4 \mathrm{can}$ be strenthened as follows.

THEOREM 3.1. Let $G$ be an annulus and let $L: \bar{G} \rightarrow 2^{\mathrm{C}^{n}}$ be a multifunction satisfying conditions of Theorem 1.4. Then, there is $W \in H^{\infty}\left(G, \mathbf{C}^{n}\right)$ such that $\tilde{W}(\varsigma) \in L(\varsigma), \varsigma \in \partial G$, a.e. $d \omega_{p}$.

Proof (SKETCH). Let $\Phi: D \rightarrow G$ be a uniformizer with $D$ equal to the unit disc. Let $L_{1}(z)=L(\Phi(z)), z \in D$. To extend $L_{1}$ to $\partial D$, recall, that $\Phi$ can be actually defined in $\bar{D} \backslash\{p, q\}$, where $p, q$ are two points, so that $\Phi: \bar{D} \backslash\{p, q\} \rightarrow \bar{G}$ is continuous. Let

$$
\begin{aligned}
& L_{1}(\zeta)=L(\Phi(\varsigma)), \quad \varsigma \in \partial D \backslash\{p, q\}, \\
& L_{1}(\varsigma)=\operatorname{co}\left(\bigcup_{\xi \in \partial G} L(\xi)\right), \quad \varsigma=p, q .
\end{aligned}
$$

Then, $L_{1}: \bar{D} \rightarrow 2^{\mathbf{C}^{n}}$ is an upper semicontinuous multifunction with convex sections, which is analytic in $D$ by Proposition 2.2 .

Consider the polynomially convex hull $Y$ of the set $X=\{(\varsigma, w) ; \varsigma \in \partial D, w \in$ $\left.L_{1}(\varsigma)\right\}$, and let $Y(z)=\left\{w \in \mathbf{C}^{n}:(z, w) \in Y\right\}$. By a simple argument, like in [17, Lemma 5(i)], $Y \cap\left(\partial D \times \mathbf{C}^{n}\right)=X$, that is $Y(\varsigma)=L_{1}(\varsigma), \varsigma \in \partial D$. By the special case $k=0$ of Theorem 1.4 (or by $[\mathbf{1}, \mathbf{1 7}]$ ), there exists $g \in H^{\infty}\left(D, \mathbf{C}^{n}\right)$, such that $\tilde{g}(\zeta) \in L_{1}(\zeta)$ a.e. $d \omega_{p}$. By the maximum property, the graph of $g$ must be contained in the hull $Y$, that is $g(z) \in Y(z), z \in D$. Let $\left\{\gamma^{n}\right\}$ be the (cyclic) group of deck transformations. The sequence of averages

$$
g_{N}(z)=(2 N+1)^{-1} \sum_{i=-N}^{N} g\left(\gamma^{j}(z)\right)
$$

has a subsequence convergent to a function $g_{\infty} \in H^{\infty}\left(G, \mathbf{C}^{n}\right)$, which is invariant with respect to $\left\{\gamma^{n}\right\}$, where $W \in H^{\infty}(G)$, cf. $[\mathbf{9}, \S 1]$. 
On the other hand, the $Y(z)$ have to be convex (see [1, Corollary] or Corollary 3.4 below); therefore $g_{N}(z) \in Y(z), N=1,2, \ldots, z \in D$, and so $g_{\infty}(z) \in Y(z)$. Since $Y(\cdot)$ is upper semicontinuous on $\bar{D}, \tilde{g}\left(e^{i \theta}\right) \in Y\left(e^{i \theta}\right)=L\left(\Phi\left(e^{i \theta}\right)\right)$ a.e. $d \theta$. Almost everywhere $\Phi^{\prime}\left(e^{i \theta}\right) \neq 0$; therefore $\tilde{g}\left(e^{i \theta}\right)=\tilde{W} \circ \Phi\left(e^{i \theta}\right)$ a.e. $d \theta$, and so $\tilde{W}(\zeta) \in L(\varsigma)$ a.e. Q.E.D.

Although we can obtain a regular selection $W(\zeta)$ in the above theorem, it is not possible to prescribe the value $W\left(z^{*}\right)$, as an arbitrary element $w^{*} \in L\left(z^{*}\right)$. (This is in contrast with the case of the unit disc, see $[\mathbf{1}$ and 17].) Before we give an example to this effect, we discuss some auxiliary facts.

Proposition 3.2. Let $G$ be as in Theorem 1.4 and let $X \subset \partial G \times \mathbf{C}^{n}$ be a compact subset with nonempty and polynomially convex sections $X(\varsigma)=\{w \in$ $\left.\mathbf{C}^{n}:(\zeta, w) \in X\right\}, \zeta \in \partial G$. Fix points $a_{1}, \ldots, a_{k}$, one in each bounded component of $\mathbf{C} \backslash G$. Assume, that the hull $Y$ of $X$, with respect to the algebra

$$
A=P\left[z, w_{1}, \ldots, w_{n},\left(z-a_{1}\right)^{-1}, \ldots,\left(z-a_{k}\right)^{-1}\right],
$$

is not contained in $\partial G \times \mathbf{C}^{n}$. Then $Y$ is equal to the graph of maximal multifunction $L: G \rightarrow 2^{\mathbf{C}^{n}}$, which is upper semicontinuous in $\bar{G}$, analytic in $G$, and satisfies

$$
L(\varsigma) \subset X(\varsigma), \quad \varsigma \in \partial G \text {. }
$$

PROOF (SKETCH). The multifunction $z \rightarrow Y(z): G \rightarrow 2^{\mathrm{C}^{n}}$ is analytic by Rossi's local maximum modulus principle ( $Y$ being the Gelfand space of $A$ ), and is clearly upper semicontinuous. On the other hand, the graph of each multifunction $L$ under consideration has the local maximum property and satisfies (3.1), and so, it must be contained in the hull $Y$. Q.E.D.

Proposition 3.3. Let $K: G \rightarrow 2^{\mathrm{C}^{n}}$ be an analytic multifunction and let $L(z)$, $z \in G$, be the convex hull of $K(z)$. Then $L: G \rightarrow 2^{\mathrm{C}^{n}}$ is an analytic multifunction. (See $[\mathbf{1 5 a}, \S 7 . \mathrm{C}(\mathrm{b})]$.

The next corollary follows immediately by combining Propositions 3.2 and 3.3.

COROLlary 3.4. Under assumptions of Proposition 3.2, if all the boundary sections $X(\zeta), \zeta \in \partial G$, are convex, then all the sections $Y(z), z \in G$, of the rational hull $Y$ (in the sense of Proposition 3.2) of $X$ are convex as well.

EXAMPLE 3.5. Let $G=\{z \in \mathbf{C}: r<|z|<1\}, 0<r<1$, and let $X=\{(z, w) \in$ $\mathbf{C}^{2}:|z|=r$ or $\left.1,|w|=|z|^{\alpha}\right\}$, where $\alpha$ is a fixed noninteger real number. Let $Y$ be the rational hull of $X$ (in the sense of Proposition 3.2). Then $Y \backslash X$ cannot be covered by graphs of single-valued analytic functions.

PROOF. Apply first Proposition 3.2 to show that

$$
Y\left\{(z, w) \in \mathbf{C}^{2}: r \leq|z| \leq 1,|w| \leq|z|^{\alpha}\right\} .
$$

Clearly, $Y \backslash X$ has the local maximum property, so $z \rightarrow Y(z): G \rightarrow 2^{\mathrm{C}}$ is analytic. Consider any multifunction $L$ with properties as in Proposition 3.2, in particular (3.1). The function

$$
\psi(z)=\max \{\log |w|: w \in L(z)\}
$$

is subharmonic in $G$ by $[\mathbf{1 8}$, Proposition 3.4 or 15, Theorem 3.2]. Since $\psi(\varsigma) \leq$ $\alpha \log |\zeta|$ for $\zeta \in \partial G, \psi(z) \leq \alpha \log |z|$ in $G$, so $L(z) \subset Y(z), z \in G$. By Proposition $3.2, Y$ is the (rational) hull of $X$. 
Now fix $z^{*} \in G$ and $w^{*}$ with $\left|w^{*}\right|=\left|z^{*}\right|^{\alpha}$, and suppose there exists $f \in H^{\infty}(G)$ such that $f(z) \in Y(z), z \in G$, and $f\left(z^{*}\right)=w^{*}$. Then, $\log |f(z)|-\alpha \log |z| \leq 0$, $z \in G$, with equality at $z=z^{*}$, which implies $\log |f(z)| \equiv \alpha \log |z|$ in $G$. If $\alpha$ is not an integer, such $f$ does not exist. Q.E.D.

REMARK 3.6. Using methods of this section, one can prove that if $Y$ is the rational hull of $X$ as in Proposition 3.2 and sections $X(\varsigma), \varsigma \in G$, are convex, then $Y \backslash X$ can be covered by graphs of many-valued analytic functions $f: G \rightarrow \mathbf{C}$ (i.e. by immersed Riemann surfaces). For the proof one has to consider the composition $L_{1}(z)=Y \circ \Phi(z)$, where $\Phi: D \rightarrow G$ is a uniformizer as in the proof of Theorem 3.1 , and to observe that $L_{1}(\cdot)$ is the maximal analytic multifunction with boundary values $X \circ \Phi(\zeta)$ (additional definition is needed at points of $\partial D \backslash \Phi^{-1}(\partial G)$ ). Then apply $[\mathbf{1}$ or $\mathbf{1 7}]$ to get a selection $h$ of $L_{1} ; h \circ \Phi^{-1}$ will be a desired many-valued selection. We omit further details.

REMARKS. The averaging argument in the proof of Theorem 3.1 (which we took from Jones and Marshall [9]) is due to Scheinberg [14] and Stout [19].

Maximal analytic multifunctions satisfying condition (3.1) were studied by Ransford [11, pp. 497-499].

Proposition 3.2 was observed independently by J. Wermer and the author.

4. The generalized corona problem for ideals. Consider $f_{1}, \ldots, f_{n}, g \in$ $H^{\infty}(G), G \subset \mathbf{C}$, such that

$$
|g(z)|^{2} \leq\left|f_{1}(z)\right|^{2}+\left|f_{2}(z)\right|^{2}+\cdots+\left|f_{n}(z)\right|^{2} \leq 1, \quad z \in G
$$

The problem whether, under these assumptions, $g$ must belong to the ideal $A f_{1}+$ $\cdots+A f_{n}$ (further denoted by $J\left(f_{1}, \ldots, f_{n}\right)$ ), was posed and answered negatively (in the case of the unit disc) by Rao [10] (see also Garnett [6, VIII, Ex. 3, p. 369]). A positive result was contributed by $\mathrm{T}$. Wolff, who has shown (in the case of the unit disc), that $g^{3} \in J\left(f_{1}, \ldots, f_{n}\right)$. In this section, we prove the next two facts, which generalize (and slightly improve) Wolff's theorem.

THEOREM 4.1. Let $G$ be a domain regular in the sense of potential theory (of arbitrary connectivity) such that the sequence of critical points is interpolating for $H^{\infty}(G)$. Then

(a) $g^{4}$ belongs to $J\left(f_{1}, \ldots, f_{n}\right)$, whenever (4.1) holds for $f_{1}, \ldots, f_{n}, g \in H^{\infty}(G)$.

(b) Moreover, $g^{3+\varepsilon} \in J\left(f_{1}, \ldots, f_{n}\right)$ provided $g^{\varepsilon}$ is defined, $\varepsilon>0$.

THEOREM 4.2. If $G$ is a finitely connected domain as in Theorem 1.4, then

(a) $g^{3} \in J\left(f_{1}, \ldots, f_{n}\right)$, whenever $f_{1}, \ldots, f_{n}, g$ belong to $H^{\infty}(G)$ and satisfy (4.1).

(b) Moreover, $g^{2+\varepsilon} \in J\left(f_{1}, \ldots, f_{n}\right)$, provided $g^{\varepsilon}$ is defined and $f=\left(f_{1}, \ldots, f_{n}\right)$ does not vanish at any of the critical points $z_{1}^{*}, \ldots, z_{k}^{*}$.

The use of the fractional power $g^{\varepsilon}$ is, of course, best motivated if $g$ is a nowhere vanishing function in the unit disc. In the next section, we will give an example of such $g$ and of $f_{1}, f_{2} \in H^{\infty}(D)$, satisfying (4.1), such that $g^{2-\varepsilon}$ does not belong to $J\left(f_{1}, f_{2}\right)$ for any positive $\varepsilon$. The same problem for $g^{2}$ seems to be open.

We need the following improvement of Theorem 2.3.

LEMMA 4.3. For every positive $\alpha$ and for every real a, there exists a constant $C_{\alpha}=C(\alpha, a)$ and a continuous multifunction $K:\left\{z \in \mathbf{C}^{n}:|z| \geq e^{a}\right\} \rightarrow 2^{\mathbf{C}^{n}}$, which 
is analytic in $\left\{|z|>e^{a}\right\}$, and such that

$$
\begin{aligned}
& \operatorname{gr}(K) \subset\left\{(z, w) \in \mathbf{C}^{n} \times \mathbf{C}^{n}:\langle z, w\rangle=1\right\} \\
& \max |K(z)|:=\max \{|w|: w \in K(z)\} \leq C_{\alpha}|z|^{1+\alpha}, \quad|z| \geq e^{a} .
\end{aligned}
$$

PROOF. It is enough to prove the lemma for $a=0$. (The notion of an analytic multifunction is clearly independent with respect to change of variables.) By $[\mathbf{1 8}$, Lemma 1.1], the multifunction

$$
K(z)=\left\{w \in \mathbf{C}^{n}:\langle z, w\rangle=1,\left.|w-| z\right|^{-2} \bar{z} \mid \leq e^{\rho(\log |z|)}\right\},
$$

where $\rho:(0, \infty)$ is a smooth function, is analytic in $\{|z|>1\}$, if $\rho$ is convex in $(0, \infty)$, and the inequality

$$
\rho^{\prime \prime}(x)\left(e^{2 \rho(x)+2 x}\left(\rho^{\prime}(x)-1\right)-2\right)>2\left(\rho^{\prime}(x)+1\right)^{2}
$$

holds. (With obtuse inequality, (4.5) is also a necessary and sufficient condition, assuming convexity of $\rho$.) After substituting $\rho(t)=\frac{1}{2} f(4 t)-t$, the inequality reduces to

$$
f^{\prime \prime}\left(e^{f}\left(f^{\prime}-1\right)-1\right)>\frac{1}{2}\left(f^{\prime}\right)^{2} .
$$

This observation, the equation

$$
\max |K(z)|=|z|^{-1}\left(e^{f(4 \log |z|)}+1\right)^{1 / 2},
$$

and some simple computation show quickly that it suffices to check the following Assertion in order to obtain (4.3).

Assertion. For every positive $\alpha$, there exist positive constants $r, b, c$ such that the convex function

$$
f(x)=2 R+(1+\alpha / 2) x+b e^{-c x}
$$

satisfies $(4.6)$ in $(0, \infty)$.

Substituting (4.8) in (4.6), we obtain

$$
\begin{aligned}
\text { LHS } & =f^{\prime \prime}\left(e^{f}\left(f^{\prime}-1\right)-1\right) \\
& =b c^{2}\left(e^{2 R} \exp \left(b e^{-c x}\right) \exp ((1+\alpha / 2-c) x)\left(\alpha / 2-b c e^{-c x}\right)-e^{-c x}\right) ; \\
\text { RHS } & =\frac{1}{2}\left(1+\alpha / 2-b c e^{-c x}\right)^{2} .
\end{aligned}
$$

We specify now $c=1+\alpha / 2, b=\alpha /(4+2 \alpha)$. Note, that $\alpha / 2-b c e^{-c x} \leq \alpha / 4$, $\exp \left(b e^{-c x}\right) \geq 1$, and $e^{-c x} \leq 1$. Then

$$
\text { LHS } \geq(\alpha / 4)(1+\alpha / 2)\left(e^{2 R}(\alpha / 4)-1\right) ; \quad \text { RHS } \leq \frac{1}{2}(1+\alpha / 2)^{2} .
$$

This implies that the Assertion holds if $R \geq \frac{1}{2} \log (1 / \alpha)+\frac{1}{2} \log (1+1 / \alpha)+\log 2$. Using this, we can obtain the following estimate for $C_{\alpha}$ in (4.3):

$$
C_{\alpha} \leq((4 / \alpha)(1+1 / \alpha)+1)^{1 / 2} \text {. Q.E.D. }
$$

COROLlaRY 4.4. Let $G$ be a finitely connected domain as in Theorem 1.4. Let $f=\left(f_{1}, \ldots, f_{n}\right) \in H^{\infty}\left(G, \mathbf{C}^{n}\right), g \in H^{\infty}(G)$. Assume, that $|g(z)| \leq|f(z)|, z \in G$, 
and, that for given $\varepsilon>0, g^{\varepsilon}$ is defined. Then there exists a meromorphic vectorvalued function $W=\left(W_{1}, \ldots, W_{n}\right)$ with simple poles at critical points $z_{1}^{*}, \ldots, z_{k}^{*}$ and nontangential boundary values $\tilde{W}(\varsigma)$ a.e. $d \omega_{p}$ such that

$$
\begin{aligned}
& g^{2+\varepsilon}(z)=\sum_{i=1}^{n} W_{i}(z) f_{i}(z), \quad z \in G \backslash\left\{z_{1}^{*}, \ldots, z_{k}^{*}\right\}, \\
& |\tilde{W}(\varsigma)| \leq C_{\varepsilon}, \quad \text { a.e. } d \omega_{p},
\end{aligned}
$$

where $C_{\varepsilon}$ depends only on $\varepsilon$.

Proof. We can assume, without loss of generality, that $|g(z)|<|f(z)|$ whenever $f(z) \neq 0$. (Replace $g$ by $(1-\delta) g, \delta>0$, if necessary.) Consider the set-valued function $K:\left\{u \in \mathbf{C}^{n}:|u| \geq 1\right\} \rightarrow 2^{\mathbf{C}^{n}}$, satisfying the conditions of Lemma 4.3, with $\alpha<\varepsilon$. By Remark 2.4, we may assume, that $f, g$ are continuous on $\bar{G}$ as well. Then, the composition $R(z)=K\left(g(z)^{-1} f(z)\right)$ is well defined and upper semicontinuous in $\bar{G} \backslash g^{-1}(0)$, and analytic in $G \backslash g^{-1}(0)$ by Proposition 2.2. Moreover, by (4.3),

$$
\max |R(z)| \leq C_{\alpha}|g(z)|^{-1-\alpha} .
$$

Now let

$$
L(z)= \begin{cases}g(z)^{1+\varepsilon} R(z), & \text { if } z \in \bar{G}, g(z) \neq 0, \\ \{0\}, & \text { if } g(z)=0 .\end{cases}
$$

Clearly, $L$ is upper semicontinuous in $\bar{G} \backslash g^{-1}(0)$; by (4.12), it is also upper semicontinuous at the points of $g^{-1}(0)$. By $[\mathbf{1 5}$, Proposition 5.1], the multifunction $L$ is analytic in $G \backslash g^{-1}(0)$. We will show now that $L$ is also analytic in $G$. By [18, Definition 0.2 and Proposition 3.4], we have to check that if $h(z, w)$ is an analytic function in a neighborhood of $\left(z^{*}, 0\right), g\left(z^{*}\right)=0$, then $|h| \mid \operatorname{gr}(L)$ cannot have a strict local maximum at $\left(z^{*}, 0\right)$. Indeed, by [18, Proposition 3.4], the function $v(z)=\max \{|g(z, w)|: w \in L(z)\}$, which is continuous near $z^{*}$, is subharmonic in a pointed neighborhood of $z^{*}$ and so subharmonic in the full neighborhood (by the well-known results about removable singularities for subharmonic functions). Note also, that

$$
\max |L(z)| \leq C_{\alpha}|g(z)|^{\varepsilon-\alpha} \leq C_{\alpha} .
$$

Applying Theorem 1.4 to $L$, we obtain the desired meromorphic function $W(\cdot)$ such that $\tilde{W}(\varsigma) \in L(\varsigma)$ a.e. $d \omega_{p}$. It follows from the definition of $L(\cdot)$ and properties of $K(\cdot)$ that $\left\langle g(\zeta)^{-1-\varepsilon} \tilde{W}(\zeta), g(\zeta)^{-1} \tilde{f}(\zeta)\right\rangle=1$, that is $g(\zeta)^{2+\varepsilon}=\langle\tilde{W}(\zeta), \tilde{f}(\zeta)\rangle$ a.e. $d \omega_{p}$, which implies (4.10). We obtain (4.11) by (4.13). Q.E.D.

PROOF OF THEOREM 4.1. We will find $g_{1}, \ldots, g_{n} \in H^{\infty}$ such that

$$
\sum_{i} f_{i} g_{i} \equiv g^{3+\varepsilon} \text {. }
$$

Since we will obtain uniform estimates on $g_{1}, \ldots, g_{n}$, we can assume, by Remark 2.4 , that $G$ is finitely connected and has smooth boundary. Let $M$ denote the interpolation constant for the sequence of critical points $z_{1}^{*}, \ldots, z_{k}^{*}$. We can find $k=\left(k_{1}, \ldots, k_{n}\right) \in H^{\infty}\left(G, \mathbf{C}^{n}\right)$ such that $|k(z)| \leq M$ and $g\left(z_{j}^{*}\right)=\left\langle k\left(z_{j}^{*}\right), f\left(z_{j}^{*}\right)\right\rangle$. Then

$$
g=\sum_{i} k_{i} f_{i}+P q, \quad \text { where }\|P q\|_{\infty} \leq M+1,
$$


and $P(\cdot)$ is defined by $(1.2)$, and $q \in H^{\infty}(G)$. Let $W=\left(W_{1}, \ldots, W_{n}\right)$ be as in Corollary 4.4. Then

$$
g^{3+\varepsilon}=\sum_{i}\left(g^{2+\varepsilon} k_{i}\right) f_{i}+g^{2+\varepsilon} P q=\sum_{i}\left(g^{2+\varepsilon} k_{i}\right) f_{i}+\sum_{i} W_{i} f_{i} P q,
$$

for $z \in G \backslash\left\{z_{1}^{*}, \ldots, z_{k}^{*}\right\}$. Hence, the functions $g_{i}=g^{2+\varepsilon} k_{i}+\left(W_{i} P\right) q$, which are holomorphic in $G$, satisfy (4.14). Furthermore, by (4.11), the following uniform estimate holds:

$$
\sup \left(\sum_{i}\left|g_{i}(z)\right|^{2}\right)^{1 / 2} \leq \sup \operatorname{ess}|k(\varsigma)|+(\sup \operatorname{ess}|\tilde{W}(\varsigma)|)\|P q\|_{\infty} \leq M+C_{\varepsilon}(M+1) \text {. }
$$

Q.E.D.

ProOF OF THEOREM 4.2. (b) Since $f\left(z_{j}^{*}\right)=0, j=1, \ldots, k$, we can find $k_{1}, \ldots, k_{n} \in H^{\infty}(G)$ such that $1=\sum_{i} k_{i} f_{i}+P q$, for some $q \in H^{\infty}(G)$. Then

$$
g^{2+\varepsilon}=\sum_{i}\left(k_{i} g^{2+\varepsilon}\right) f_{i}+g^{2+\varepsilon}(P q)
$$

Now, the right side of this equation can be represented by $\sum_{i} f_{i}\left(k_{i} g^{2+\varepsilon}+W_{i} P q\right)$ in the same way as it was done in (4.15) in the last proof.

(a) Choose a function $P^{*}$ in $H^{\infty}(G)$, continuous on $\bar{G}$ and such that

$$
\left(P^{*}\right)^{-1}(0)=f^{-1}(0) \cap\left\{z_{1}^{*}, \ldots, z_{k}^{*}\right\}, \quad \inf _{\varsigma \in \partial G}\left|P^{*}(\varsigma)\right| \geq 1,
$$

and the corresponding zeros are of the same multiplicity. Then we can represent $f=f^{*} P^{*}$, with $f^{*}\left(z_{j}^{*}\right) \neq 0, j=1, \ldots, k$, and $f^{*} \in H^{\infty}(G)$. Since $|g(z)| \leq|f(z)|$, we obtain in the same way that $g=P^{*} g^{*}$, where $g^{*} \in H^{\infty}(G)$ and $\left|g^{*}(z)\right| \leq$ $\left|f^{*}(z)\right| \leq 1, z \in G$.

By Corollary 4.4, there exists a meromorphic function $W=\left(W_{1}, \ldots, W_{n}\right) \in$ $H^{\infty}\left(G, \mathbf{C}^{n}\right)$, with poles at $z_{1}^{*}, \ldots, z_{k}^{*}$, such that

$$
\left(g^{*}\right)^{3}=\sum_{i} W_{i} f_{i} \quad \text { in } G \backslash\left\{z_{1}^{*}, \ldots, z_{k}^{*}\right\} .
$$

Since $f^{*}(z)$ does not vanish at critical points, we can find $q, k_{1}, \ldots, k_{n} \in H^{\infty}(G)$, so that

$$
1=\sum_{i} f_{i}^{*}(z) k_{i}(z)+P(z) q(z)
$$

Multiplying the last equation by $g^{3}$, we obtain

$$
g^{3}=\sum_{i} f_{i}\left(k_{i}\left(P^{*}\right)^{2}\right)+\left(g^{*}\right)^{3} P(z)\left(P(z)^{*}\right)^{3} q(z) .
$$

Substituting in the last formula $\left(g^{*}\right)^{3}$ given by (4.16), we obtain that functions $g_{i}=k_{i}\left(P^{*}\right)^{3}+\left(W_{i} P\right)\left(P^{*}\right)^{3} q$ are bounded holomorphic solutions to the equation $g^{3}=\sum_{i} f_{i} g_{i}$. Q.E.D.

REMARKS. Our methods differ from those of T. Wolff (neither the $\bar{\partial}$-equation, nor the Koszul complex are used), cf. Garnett [6, Theorem 2.3]. In particular, the proof of Corollary 4.4, when specified in the case of the unit disc, gives the new proof of Wolff's theorem. 
The proofs of Theorems 4.1 and 4.2 extend to Riemann surfaces (finitely connected in the latter case), cf. remark after the proof of Theorem 2.1.

Note added July 16, 1986. Theorems 4.1 and 4.2 can also be obtained from the results of Jones and Marshall $[\mathbf{9}, \S 2]$. In particular, Lemma 2.2 in [9] allows for transferring the problem to the unit disc. In addition, under the (apparently stronger) assumptions of Theorem 2.10 in [9] the exponents 4 and $3+\varepsilon$ can be lowered to 3 and $2+\varepsilon$ respectively.

The author is grateful to Professor Peter Jones for these observations.

5. Estimates and examples. In this section, we will estimate (in the case of the unit disc) some of the constants and parameters, considered previously. This will give some indications of how distant results presented here are from the optimal ones.

First consider the best corona constant $K_{\delta}$ - that is, the smallest $K$ such that, whenever $\delta^{2} \leq\left|f_{1}(z)\right|^{2}+\cdots+\left|f_{n}(z)\right|^{2} \leq 1, f_{1}, \ldots, f_{n} \in H^{\infty}(D)$, then the solutions $g_{1}, \ldots, g_{n} \in H^{\infty}(D), g_{1} f_{1}+\cdots+g_{n} f_{n}=1$ can be found so that $\left|g_{1}(z)\right|^{2}+\cdots+$ $\left|g_{n}(z)\right|^{2} \leq K^{2}$.

By $[18, \S 2]$ or the proof of Theorem 2.1 above, $K_{\delta} \leq C_{\delta}=C_{\delta, 1}$, where $C_{\delta, 1}$ is as in Theorem 2.3. By the construction of the multifunction $K(\cdot)$ in this theorem, as discussed in the proof of Lemma 4.3 , and by (4.7),

$$
\left.C_{\delta} \leq \max _{x \in[4 \log \delta, 0]} e^{-x} e^{f_{1}(x)}+1\right)^{1 / 2},
$$

where $f_{1}(\cdot)$ is a solution to $(4.6)$ in $[4 \log \delta, 0]$. We can take e.g. $f_{1}(x)=f(x-4 \log \delta)$, where $f$ is any solution to $(4.6)$ in $[0, \infty)$. Hence, $C_{\delta}^{2} \leq 1+\exp (f(4 \log (1 / \delta)))$, and by the proof of Lemma 4.3, Assertion,

$$
\begin{aligned}
f\left(4 \log \left(\frac{1}{\delta}\right)\right. & \leq 2 \log 2+\log \left(\frac{1}{\alpha}\right)+\log \left(1+\frac{1}{\alpha}\right)+\left(1+\frac{\alpha}{2}\right) 4 \log \left(\frac{1}{\delta}\right)+\frac{\alpha}{4+2 \alpha} \\
& =2 \log 2+2 \log \left(\frac{1}{\alpha}\right)+\left(1+\frac{\alpha}{2}\right) 4 \log \left(\frac{1}{\delta}\right)+o(\alpha) .
\end{aligned}
$$

Since the sum $2 \log (1 / \alpha)+(1+\alpha / 2) 4 \log (1 / \delta)$ has minimum at $\alpha=(\log (1 / \delta))^{-1}$, we obtain

$$
f\left(4 \log \left(\frac{1}{\delta}\right)\right)=2 \log 2+2+4 \log \left(\frac{1}{\delta}\right)+2 \log \log \left(\frac{1}{\delta}\right)+o(\delta),
$$

and so $C_{\delta}^{2} \leq 1+4 e^{2}(1 / \delta)^{4}(\log (1 / \delta))^{2} \exp (o(\delta))$.

COROLlaRY 5.1. $K_{\delta} \leq C_{\delta}, \delta>0$, where $K_{\delta}$ denotes the corona constant and $C_{\delta}$ (= the best constant $C_{\delta, 1}$ in (2.4)) satisfies

$$
\limsup _{\delta \rightarrow 0} \frac{C_{\delta}}{(1 / \delta)^{2} \log (1 / \delta)} \leq 2 e .
$$

REMARK. The same rate of growth for $K_{\delta}$ was obtained by different methods by Berndtsson and Ransford [2, $\S 2]$ (in case of two generators).

The precise rate of growth of $K_{\delta}$ is unknown. Below, we modify the counterexample, due to A. M. Gleason (cf. Garnett [6, VII, p. 293]), to provide the following estimate. 
Proposition 5.2. $K_{\delta} \geq(1 / 4 \sqrt{2}) \delta^{-2}, \delta>0$.

ProOF. For every $C \in(1, \infty)$ and for every integer $N$, consider the corona data in the unit disc:

$$
f_{1}(z)=\frac{1}{\sqrt{2}} z^{N+1}, \quad f_{2}(z)=\frac{1}{\sqrt{2}} \frac{z^{N}-r^{N}}{1-r^{N} z^{N}}, \quad|z|<1,
$$

where $r^{N}=1 / C$. Clearly, $\left|f_{1}(z)\right|^{2}+\left|f_{2}(z)\right|^{2} \leq 1$; let $\delta_{N}$ denote the maximal $\delta$, such that $\delta^{2} \leq\left|f_{1}(z)\right|^{2}+\left|f_{2}(z)\right|^{2}, z \in D$. It turns out, that

$$
\text { if } g_{1} f_{1}+g_{2} f_{2} \equiv 1 \text { in } D \text {, then }\left\|g_{1}\right\|_{\infty} \geq \sqrt{2} C^{2} \text {. }
$$

To see this, denote by $r \omega_{0}, r \omega_{1}, \ldots, r \omega_{N-1}$ the roots of the Blaschke product $\sqrt{2} f_{2}$ (where $\left|\omega_{i}\right|=1$ ). Observe that, if $1 \equiv g_{1} f_{1}+g_{2} f_{2}$, then $1=g_{1}\left(r \omega_{i}\right) f_{1}\left(r \omega_{i}\right)=$ $(1 / \sqrt{2}) r^{N+1} \omega_{i} g_{1}\left(r \omega_{i}\right)$, and so the function $h(z)=(1 / \sqrt{2}) r^{N+1} g(z), z \in D$, solves the interpolation problem $h\left(r \omega_{i}\right)=\omega_{i}^{-1}, i=0,1, \ldots, N-1$. It is shown in Garnett $\left[6\right.$, p. 294] that for any such solution $h,\|h\|_{\infty} \geq r^{1-N}$, i.e. $\left\|(1 / \sqrt{2}) r^{N+1} g\right\|_{\infty} \geq$ $r^{1-N}$, which shows (5.1).

We have to estimate now the constants $\delta_{N}$. Clearly,

$$
\delta_{N} \geq \varepsilon_{N}:=\min _{|z| \leq 1}\left(\max \left|f_{1}(z)\right|,\left|f_{2}(z)\right|\right) .
$$

Since

$$
\min _{|z|=t}\left|f_{2}(z)\right|=\frac{(1 / \sqrt{2})\left|t^{N}-r^{N}\right|}{\left|1-r^{N} t^{N}\right|}
$$

and $\left|z^{N+1}\right|=t^{N+1}$ on $|z|=t$, we substitute $s=t^{N}$ and get $\varepsilon_{N}=\min _{s \in[0,1]} f_{N}(s)$, where

$$
f_{N}(s)=(1 / \sqrt{2}) \max \left(s^{1+1 / N},|s-1 / C| /|1-s / C|\right), \quad 0 \leq s \leq 1 .
$$

Since this nondecreasing sequence of functions is uniformly convergent, $\lim \varepsilon_{N}=$ $\inf f(s)$, where

$$
f(s)=(1 / \sqrt{2}) \max (s,|s-1 / C| /|1-s / C|), \quad 0 \leq s \leq 1 .
$$

Simple geometric considerations show that $f$ has the only minimum at $s$ such that $0 \leq s \leq 1 / C$ and $s=(1 / C-s) /(1-s / C)$. This yields $s=1 /\left(C+\sqrt{C^{2}-1}\right)$, so $\min f(s) \geq(2 \sqrt{2} C)^{-1}$. Therefore,

$$
\liminf _{N} \delta_{n} \geq \lim _{N} \varepsilon_{n} \geq(2 \sqrt{2} C)^{-1} .
$$

To conclude the proof, fix now $\delta \in(0,1)$ and choose $C$ so that $(2 \sqrt{2} C)^{-1}=\delta$. It follows from (5.1) and (5.2) that $K_{\delta} \geq\left(4 \sqrt{2} \delta^{2}\right)^{-1}$. Q.E.D.

EXAMPLE 5.3. We will now use the previous example to construct functions $g, f_{1}, f_{2} \in H^{\infty}(D)$ such that

$$
\begin{gathered}
0<|g(z)|^{2}<\left|f_{1}(z)\right|^{2}+\left|f_{2}(z)\right|^{2}<1, \quad z \in D, \\
g^{2-\varepsilon} \notin J\left(f_{1}, f_{2}\right), \quad 0<\varepsilon .
\end{gathered}
$$

Consider disjoint unit discs $D_{N}=\{z \in \mathbf{C}:|z-3 N|<1\}, N=1,2, \ldots$ Let $\delta_{N} \in(0,1)$ be numbers to be specified later. As in the last proof, one can find $f_{1 N}, f_{2 N} \in H^{\infty}\left(D_{N}\right)$, so that

$$
4 \delta^{2}<\left|f_{1 N}(z)\right|^{2}+\left|f_{2 N}(z)\right|^{2}<1 / 4,
$$


and whenever $g_{1} f_{1 N}+g_{2} f_{2 N} \equiv 1, g_{1}, g_{2} \in H^{\infty}\left(D_{N}\right)$, then $\left\|g_{1}\right\|_{\infty} \geq 3 c \delta_{N}^{-2}$, where $c$ is a universal constant. Consequently, holomorphic solutions $g_{1}, g_{2}$ of the equation

$$
\delta_{N}^{2-\varepsilon}=f_{1 N}(z) g_{1}(z)+f_{2 N}(z) g_{2}(z), \quad z \in D_{N},
$$

where $\varepsilon \in[1 / N, 2]$, satisfy $\left\|g_{1}\right\|_{\infty} \geq 3 c\left(1 / \delta_{N}\right)^{1 / N}$. We now use B. Cole's ideas to make one connected domain out of the disjoint discs $D_{N}$.

First, by the Montel family argument, one can select radii $r_{N}<1$ such that

$$
\max _{|z| \leq r_{N}}\left|g_{1}(z)\right| \geq 2 c\left(1 / \delta_{N}\right)^{1 / N}
$$

even if (5.4) holds only for $z \in D_{N}^{*}:=\left\{|z-3 N|<r_{N}\right\}, g_{1}, g_{2} \in H^{\infty}\left(D_{N}^{*}\right)$. Next, using a standard Banach algebra argument, one can find $\eta_{N}>0$ small enough so that

$$
\begin{aligned}
& \text { whenever } g, f_{1}, f_{2}, g_{1}, g_{2} \in H^{\infty}\left(D_{N}^{*}\right),\left\|g-\delta_{N}\right\|_{\infty} \leq \delta_{N} \eta_{N}, \\
& \left\|f_{i}-f_{i N}\right\| \leq \eta_{N}, \text { and } g^{2-\varepsilon}=f_{1} g_{1}+f_{2} g_{2}, \varepsilon \in[1 / N, 2), \\
& \text { then }\left\|g_{1}\right\|_{\infty} \geq c\left(1 / \delta_{N}\right)^{1 / N}
\end{aligned}
$$

Consider now the closed and connected set $X \subset \mathbf{C}$, obtained by joining the closed discs $\overline{D_{N}^{*}}$ by closed segments $\left[3 N+r_{N}, 3(N+1)-r_{N+1}\right]$. Define continuous functions $\eta: X \rightarrow(0, \infty)$, and $g^{*}: X \rightarrow \mathbf{C}$ by letting $\eta\left|\overline{D_{N}^{*}} \equiv \eta_{N}, g^{*}\right| \bar{D}_{N}^{*}=2 \delta_{N}$, and interpolating linearly on the connecting segments. To define $f_{1}^{*}, f_{2}^{*}: X \rightarrow \mathbf{C}$, let $f_{i}^{*}\left|\bar{D}_{i}^{*}=f_{i N}\right| \bar{D}_{N}^{*}$ and extend them continuously to the connecting segments, so that

$$
4\left|g^{*}(z)\right|^{2}<\left|f_{i}^{*}(z)\right|^{2}+\left|f_{2}^{*}(z)\right|^{2}<1 / 4
$$

Applying so-called Carleman (or tangential) approximation on $X$, we can find an open neighborhood $G$ of $X$ and holomorphic functions $f_{1}, f_{2}, g \in H^{\infty}(G)$, such that

$$
\left|g(z)-g^{*}(z)\right|<\eta(z)\left|g^{*}(z)\right|, \quad\left|f_{i}(z)-f_{i}^{*}(z)\right|<\eta(z), \quad z \in X, i=1,2 .
$$

(This can be proved by exhausting suitably the set $X$ by simply connected compact sets $X_{N}$ and applying repeatedly Mergelyan's theorem. For more general results of this kind, as well as further references, see Boivin [3].) Shrinking $G$, if necessary, we can assume that it is simply connected. If we require also that $0<\eta_{N}<\frac{1}{4} \delta_{N}$, then by (5.3), (5.6),

$$
0<|g(z)|^{2}<\left|f_{1}(z)\right|^{2}+\left|f_{2}(z)\right|^{2}<1, \quad z \in G .
$$

Suppose now, that $g_{1}, g_{2} \in H(G)$ and $g^{2-\varepsilon}=g_{1} f_{1}+g_{2} f_{2}$ in $G$, where $0<\varepsilon<2$. By (5.5), (5.6), if $N>1 / \varepsilon$, then $\left\|g_{1} \mid D_{N}^{*}\right\|_{\infty} \geq c\left(1 / \delta_{N}\right)^{1 / N}$. If $\delta_{N}$ are so chosen that $\lim \delta_{N}^{1 / N}=0$, then $g_{1} \notin H^{\infty}(G)$. Since $G$ is biholomorphically equivalent to the unit disc, the proof is complete. Q.E.D.

REMARKS. For an account of B. Cole's argument, which we followed in the last example, see Gamelin [8, pp. 49-53].

It is possible to find an exact solution of the equation $f^{\prime \prime}\left(e^{f}\left(f^{\prime}-1\right)-1\right)=\frac{1}{2}\left(f^{\prime}\right)^{2}$, but the estimate for $C_{\delta}$ in Proposition 5.2, obtained in this way, is qualitatively the same $\left(\lim \sup C_{\delta} /\left((1 / \delta)^{2} \log (1 / \delta)\right)=2 \sqrt{e}\right)$.

ACKNOWLEDGMENTS. The author was attracted to problems studied in $\S 4$ by R. Rochberg, who suggested that analytic multifunctions might be useful in this context. The author is grateful to R. Rochberg, as well as to T. W. Gamelin and J. Wermer, for their interest in this work. 


\section{REFERENCES}

1. H. Alexander and J. Wermer, Polymomial hulls with convex fibers, Math. Ann. 271 (1985), 99-109.

2. B. Berndtsson and T. J. Ransford, Analytic multifunctions, the $\bar{\partial}$-equation, and a proof of the coroma theorem, Pacific J. Math. (to appear).

3. A. Boivin, On Carleman approximation by meromorphic functions, Proc. 8th Conf. Analytic Functions, Blazejewko, Poland, August 1982 (to appear).

4. S. Fisher, Function theory on planar domains, Wiley, New York and Toronto, 1983.

5. F. Forelli, Bounded holomorphic functions and projections, Illinois J. Math. 10 (1966), 367-380.

6. J. B. Garnett, Bounded analytic functions, Academic Press, London and New York, 1981.

7. T. W. Gamelin, Localization of the corona problem, Pacific J. Math. 34 (1970), 73-81.

8. __ Uniform algebras and Jensen measures, Cambridge Univ. Press, Cambridge and New York, 1978.

9. P. W. Jones and D. E. Marshall, Critical points of Green's function, harmonic measure and the corona problem, Institut Mittag-Leffler, Report No. 2, 1984.

10. K. V. R. Rao, On a generalized corona problem, J. Analyse Math. 18 (1967), 277-278.

11. T. J. Ransford, Interpolation and extrapolation of analytic multivalued functions, Proc. London Math. Soc. (3) 50 (1985), 480-504.

12. H. L. Royden, The boundary values of analytic and harmonic functions, Math. Z. 28 (1962), 1-24.

13. W. Rudin, Analytic functions of class $H_{p}$, Trans. Amer. Math. Soc. 78 (1955), 46-66.

14. S. Scheinberg, Thesis, Princeton Univ., 1962.

15. Z. Slodkowski, Analytic set-valued functions and spectra, Math. Ann. 256 (1981), 363-386.

15a. , Analytic set-valued functions and spectra, Inst. of Math., Polish Academy of Sciences, Preprint, October 1980.

16. __ Analytic multifunctions, q-phurisubharmonic functions and uniform algebras, Proc. Conf. Banach Algebra and Several Complex Variables, (F. Greenleaf and D. Gulick, Editors), Contemp. Math., vol. 32, Amer. Math. Soc., Providence, R. I., 1984, pp. 243-258.

17. __ Polynomial hulls with convex sections and interpolating spaces, Proc. Amer. Math. Soc. 96 (1986), 255-260.

18. _ An analytic set-valued selection and its applications to the corona theorem, to polymomial hulls and joint spectra, Trans. Amer. Math. Soc. 294 (1986), 367-377.

19. E. L. Stout, Bounded holomorphic functions on finite Riemann surfaces, Trans. Amer. Math. Soc. 120 (1965), 225-285.

20. J. Wermer, Subalgebras of the algebra of all complex-valued analytic functions on the circle, Amer. J. Math. 76 (1956), 225-242.

Department of Mathematics, University of Illinois at Chicago, Chicago, ILLINOIS 60680 